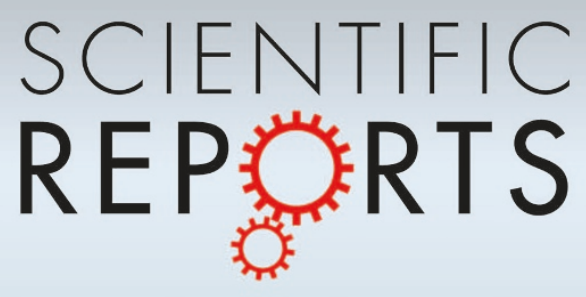

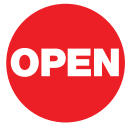

SUBJECT AREAS:

MICROWAVE PHOTONICS

NONLINEAR OPTICS

FIBRE OPTICS AND OPTICAL COMMUNICATIONS

ELECTRICAL AND ELECTRONIC ENGINEERING

Received

11 January 2013

Accepted

20 March 2013

Published

3 April 2013

Correspondence and requests for materials should be addressed to M.S. (marco. santagiustina@unipd.

\title{
All-optical signal processing using dynamic Brillouin gratings
}

\author{
Marco Santagiustina', Sanghoon Chin ${ }^{2}$, Nicolay Primerov ${ }^{2}$, Leonora Ursini' \& Luc Thévenaz ${ }^{2}$ \\ 'Department of Information Engineering, University of Padova, Italy, ${ }^{2}$ Ecole Polytechnique Fédérale de Lausanne, Switzerland.
}

The manipulation of dynamic Brillouin gratings in optical fibers is demonstrated to be an extremely flexible technique to achieve, with a single experimental setup, several all-optical signal processing functions. In particular, all-optical time differentiation, time integration and true time reversal are theoretically predicted, and then numerically and experimentally demonstrated. The technique can be exploited to process both photonic and ultra-wide band microwave signals, so enabling many applications in photonics and in radio science.

he invention and development of fiber optics represented a milestone for communications, clearly certified by the Nobel price attributed to C. Kao in 2009. There are also no doubts about the depth of the societal implications, with Internet being the most important, and the large horizons opened by optical fibers, that highly contributed to transform optics into photonics, the science of photon manipulation.

Besides the transmission, photonics also entails the all-optical signal processing techniques. However, on this regard, it is evident that fiber optics, and more in general photonics, lags well behind electronics, which is ubiquitous in information technology and also in the optical networks. It is still an open question for research to extrapolate, from the present and the medium term evolution of the state-of-art in photonics, how all-optical processing might become competitive with electronics in terms of compactness, energy consumption, integration and reliability. Nonetheless, photonics has yielded, and will continue to, several processing techniques that, being based on the fundamental properties of light, have no similar counterparts in electronics. Quantum communications are a very good example on this regard.

In this article, some unique processing properties of fiber optics, realizing functionalities more typically associated to digital electronics or, even, that cannot be achieved by electronics devices, are demonstrated. The processing functions presented here are based on a single nonlinear effect, stimulated Brillouin scattering (SBS), in particular on the generation and manipulation of the so-called dynamic Brillouin gratings (DBGs) ${ }^{1}$. Moreover, the processing functions are achieved by slight modifications of the same setup, that is conceptually very simple and requires a limited set of photonic devices.

SBS in optical fibers is already considered as a highly efficient and flexible process to achieve photonic functionalities like amplification ${ }^{2}$, sensing ${ }^{3}$, slow ${ }^{4}$ and stored light ${ }^{5}$, microwave-photonics ${ }^{6}$ and polarization control $^{7}$. In spite of the many achievements so far attained, the full potential of SBS in all-optical signal processing is surprisingly wider than foreseen, as this manuscript will demonstrate.

The first, basic element of any processing function is the capability of storing the information, which is indeed possible in SBS by transferring information to the long living acoustic wave $e^{5,8}$. This process is depicted in Fig. 1: a data waveform at frequency $\omega_{d}$ propagating in a fiber from $z=0$ to $z=L$ can be converted, by interacting with a counter-propagating short control pulse (write pulse) at frequency $\omega_{w}=\omega_{d}-\Omega_{B}$ (where $\Omega_{B}$ is the Brillouin frequency shift), into an acoustic wave which travels in the fiber at the sound velocity. Let us remark that the choice to set $\omega_{w}$ below $\omega_{d}$ is purely arbitrary; in fact the acoustic wave is created even if $\omega_{w}=\omega_{d}+\Omega_{B}$, changing its propagation direction. During the writing process, the data waveform energy is transferred to the acoustic wave, realizing the information storage. It is important to note that the acoustic wave generated by the interaction retains the amplitude and phase of the input waveform ${ }^{5,8}$.

The acoustic wave actually modulates the fiber refractive index, thus creating a $\mathrm{DBG}^{1}$. The stored information can be easily recovered from the DBG: in fact, a short optical signal (the read pulse) launched from the same side of the fiber used for the write pulse is backscattered by the DBG and thus generates the retrieved waveform. The process is particularly convenient in polarization maintaining fibers (PMFs); in fact, owing to its longitudinal nature the acoustic wave equally scatters all light polarizations. So the storage and the retrieval processes, when realized on two different orthogonal states of polarization (SOP) aligned to the birefringence axes ${ }^{1,9}$ of a PMF, are 

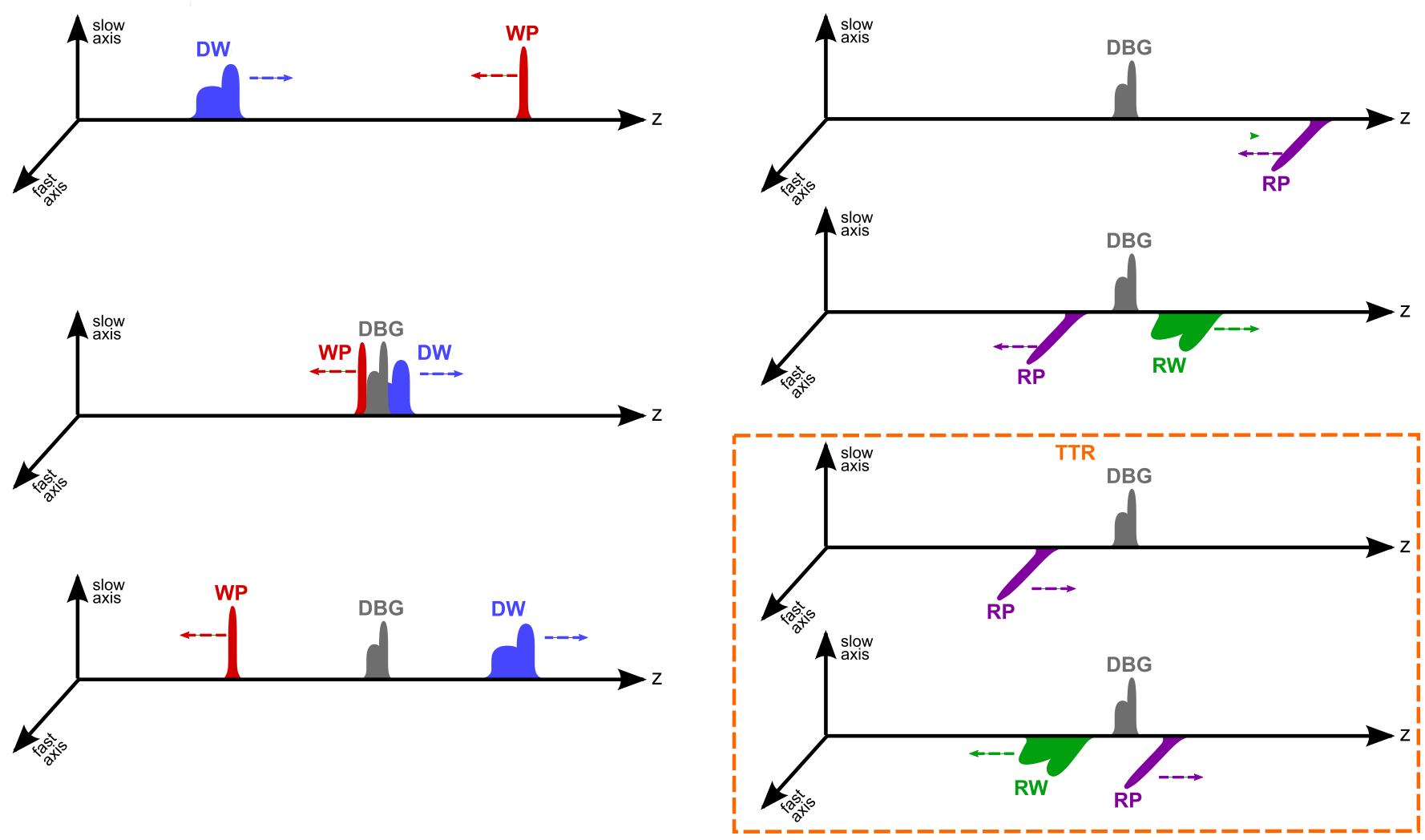

Figure $1 \mid$ Conceptual scheme of the data storage and retrieval through DBG in a PMF. The graphic within the box refers to the setup to obtain the true time reversal. DW, WP, RP and RW refer to data waveform, write pulse, read pulse and retrieved waveform, respectively.

easily decoupled in polarization, but also in frequency since the fiber birefringence entails a necessary change of the read and retrieved pulse frequencies to satisfy the phase matching.

DBGs have been already exploited to achieve breakthrough applications in sensing ${ }^{10,35}$ and to realize microwave-photonic filters ${ }^{11}$ and tunable delay lines ${ }^{12}$. Here, it is shown how stored light can be exploited to achieve unconventional all-optical signal processing functions like all-optical calculus (differentiation and integration) and true time reversal. It is remarkable that these processing functions are entirely realized in optical fibers and can be seamlessly integrated in an optical network with minor insertion losses. This is a point that makes a crucial distinction with other device-based processors.

\section{Results}

Theoretical foundations. To understand why unconventional processing is made possible by DBGs, a simple theoretical model is introduced here. The model, without loss of generality, assumes that the data waveform and write pulse are launched with SOPs aligned with the fiber slow axis while the read pulse and the retrieved waveform SOPs are aligned with the fiber fast axis. The phase matching conditions on different axis of a PMF yield a condition on the frequency of the retrieved waveform ${ }^{9}: \omega_{\text {ret }}=$ $\omega_{d}(1+\Delta n / n)$, where $\Delta n=n_{s}-n_{f}$ is the difference between the refractive indexes of the PMF slow and fast axis.

In this situation a set of equations describes the evolution of the slowly varying wave envelopes of the optical waves data waveform, read pulse, write pulse, retrieved waveform (respectively indicated by $\left.A_{d}(z, t), A_{w}(z, t), A_{r}(z, t), A_{r e t}(z, t)\right)$ and of the acoustic wave indicated by $Q$ (see the additional information document for the detailed definitions). Equations are similar to those used in ref. 9:

$$
c \partial_{z} A_{d}+\partial_{t} A_{d}=-\eta_{B} Q A_{w}+\imath \Gamma_{K}\left|A_{d}\right|^{2} A_{d}
$$

$$
\begin{gathered}
-c \partial_{z} A_{w}+\partial_{t} A_{w}=\eta_{B} Q^{*} A_{d}+\imath \Gamma_{K}\left|A_{w}\right|^{2} A_{w}, \\
c \partial_{z} A_{r e t}+\partial_{t} A_{r e t}=-\eta_{B} Q A_{r}+\imath \Gamma_{K}\left|A_{r e t}\right|^{2} A_{r e t}, \\
-c \partial_{z} A_{r}+\partial_{t} A_{r}=\eta_{B} Q^{*} A_{r e t}+\imath \Gamma_{K}\left|A_{r}\right|^{2} A_{r}, \\
\partial_{t} Q+\Gamma_{B} Q=\Gamma_{B}\left(A_{d} A_{w}^{*}+A_{r e t}^{*} A_{r}\right)+\xi .
\end{gathered}
$$

where, $c$ is the light speed in the fiber (we assumed for simplicity, but without loss of generality, that the group velocity is the same on each axis and equal to the phase velocity), $\eta_{B}$ is a normalized interaction factor (also proportional to the Brillouin gain), $\Gamma_{K}$ is proportional to the Kerr's coefficient and $\Gamma_{B}=1 / 2 \tau_{B}$ where $\tau_{B}$ is the phonon lifetime. The self phase modulation (SPM) must be taken into consideration because high power pulses are used. Finally $\xi$ represents the spontaneous Brillouin noise $(\mathrm{SpBN})^{13}$.

Writing process. Let us neglect the SPM and the SpBN in the following theoretical analysis (their effects will be considered in the additional information document). The DBGs creation can be modeled by setting $A_{r}=A_{r e t}=Q=0$ for $0 \leq z \leq L, t=0$ and the boundary conditions $A_{d}(z=0, t)=A_{d 0}(t), A_{w}(z=L, t)=A_{w L}(t)$. High fidelity data waveform storage is achieved when the write pulse is much shorter and powerful than the data waveform ${ }^{5,9}$, so that it is not depleted. Let assume the input write pulse to be an ideal Dirac function $A_{w L}(t)=\varepsilon_{w} \exp \left({ }_{l} \theta_{w}\right) \delta(t)$, where $\varepsilon_{w}=\int_{t}\left|A_{w L} d t\right|$ and $\theta_{w}$ is an arbitrary phase. The optical waves propagate without distortion, $A_{d}(z, t)=A_{d 0}(z / c-t), A_{w}(z, t)=A_{w L}[(z-L) / c+t)$, till the point of overlap, whose exact location depends on the initial delays applied to the input waveforms (for the sake of simplicity they are set to zero, so the pulses meet at $z_{c}=L / 2$ ). 
By setting $q(z, t)=Q(z, t) \exp \left(\Gamma_{B} t\right)$, Eq. (5) can be formally integrated (in time), at a fixed position $z \in\{0, L\}$ :

$$
\begin{aligned}
q(z, t) & =\Gamma_{B} \int_{0}^{t} A_{d 0}\left(\frac{z}{c}-t^{\prime}\right) A_{w L}^{*}\left(\frac{z-L}{c}+t^{\prime}\right) \exp \left(\Gamma_{B} t^{\prime}\right) d t^{\prime}= \\
& =\Gamma_{B} \varepsilon_{w} \exp \left(-\imath \theta_{w}\right) A_{d 0}\left(2 \frac{z-z_{c}}{c}\right) \exp \left(-\Gamma_{B} \frac{z-L}{c}\right) .
\end{aligned}
$$

From Eq. (6) the fundamental property of DBGs, which enables all processing functions is made clear: the wave resulting from any interaction between two waves (either optical or acoustic) is a convolution. In this case the kernel function is a Dirac function, so the stored acoustic wave retains the amplitude and phase of the input data waveform $\left(A_{d 0}\right)$. The stored data waveform is spatially compressed by a factor $2^{14,15}$ and distorted by an exponentially decaying term because the data waveform leading edge is stored for longer times with respect to the trailing edge.

If the wave processing is carried out within a few $n s$ from the storage time, the acoustic wave motion can be completely neglected and the acoustic wave decay will only decrease the amplitude of the retrieved wave.

Signal processing. In this section we present the theoretical foundation of four all-optical processing functions of the data waveform: 1) high fidelity retrieval, 2) time differentiation, 3) true time reversal, 4) time integration. The first three are all achieved by simply changing the kernel of the convolution in the retrieval process, i.e. the read pulse. As for the integration, it is easier to use a different stored waveform, as it will be shown below.

The processing of the stored signal is modeled by Eqs. (3-5); in particular for each processing function it is useful to perform a change of variables into a reference frame moving with the retrieved wave.

1) For data waveform retrieval, let us define $z^{\prime}=z-c t$ and the kernel (read pulse) is an ideal Dirac function $A_{r}(z=L, t)=A_{r L}(t)=$ $\varepsilon_{r} \exp \left(\imath \theta_{r}\right) \delta\left(t-t_{0}\right)\left(\varepsilon_{r}=\left|\int_{t} A_{r} d t\right|, \theta_{r}\right.$ arbitrary, $t_{0}$ the injection time $)$. The read pulse propagates without distortion, $A_{r}\left(z^{\prime}, t\right)=A_{r L}\left[\left(z^{\prime}-\right.\right.$ $L) / c+2 t)$, till it interacts with the acoustic wave, generating the retrieved waveform. By formally integrating Eq. (3), one gets:

$A_{r e t}\left(z^{\prime}, t\right)=-\eta_{B} \int_{0}^{t} A_{r L}\left(\frac{z^{\prime}-L}{c}+2 t^{\prime}\right) q\left(\frac{z^{\prime}}{c}+t^{\prime}\right) \exp \left(-\Gamma_{B} t^{\prime}\right) d t^{\prime},(7)$

By substituting the read pulse (Dirac distribution) and the stored acoustic wave (Eq. 6), in the reference frame fixed with the fiber $(z)$, the retrieved waveform is:

$$
A_{\text {ret }}(z, t)=A_{\text {out }} A_{d 0}\left(\frac{z}{c}-t+t_{0}\right),
$$

where $A_{\text {out }}=-\eta_{B} \Gamma_{B} \varepsilon_{w} \varepsilon_{r} \exp \left[-\Gamma_{B} t_{0}+{ }_{l}\left(\theta_{r}-\theta_{w}\right)\right]$ is a constant. The retrieved waveform is a scaled, phase shifted and delayed replica of the input data waveform, moving in the forward direction. Let us note that the exponential distortion and the compression introduced by the recording process are perfectly compensated during the reading process. Data retrieval has been experimentally demonstrated in Ref. 5, so it is not further addressed here.

2) To achieve the arbitrary waveform time differentiation the kernel (read pulse) must be the first order time derivative of a Dirac function; formally, $A_{r}(z=L, t)=A_{r L}(t)=\varepsilon_{r}^{(1)} \exp \left(\imath \theta_{r}^{(1)}\right) \delta^{(1)}\left(t-t_{0}\right)$ $\left(\varepsilon_{r}^{(1)}=\left|\int_{t}\right| A_{r L}^{(1)} d t \mid, \theta_{r}^{(1)}\right.$ arbitrary, $t_{0}$ the injection time). By considering the change of variables $z^{\prime}=z-c t$ and integrating equation (3) one gets:

$$
A_{\text {ret }}(z, t)=A_{\text {out }}^{(1)}\left[\frac{d A_{d 0}}{d t}\left(\frac{z}{c}+t_{0}-t\right)-\Gamma_{B} A_{d 0}\left(\frac{z}{c}+t_{0}-t\right)\right],
$$

where $A_{\text {out }}^{(1)}=-\eta_{B} \Gamma_{B} \varepsilon_{w} \varepsilon_{r}^{(1)} \exp \left[-\Gamma_{B} t_{0}+\imath\left(\theta_{r}^{(1)}-\theta_{w}\right)\right] / 2$. The retrieved waveform is the time differentiation of the data waveform (scaled in amplitude, phase shifted and delayed), in fact, if the time variations of the data waveform are faster than the decay time $\tau_{B}$, which is the typical experimental condition, the second term of Eq. (9) is negligible with respect to the first term. Let us remark that by repeating the process, higher order differentiation is also possible.

3) To obtain the true time reversal of the data waveform, it is necessary to exchange the roles of the read pulse and the retrieved waveform in Eqs. (3-5). The read pulse is injected from $z=0$ and propagates forward and the retrieved waveform is at frequency $\omega_{\text {ret }}$ $=\omega_{r}-\Omega_{B}$, while phase matching yields $\omega_{r}=\omega_{d}(1+\Delta n / n)$. In this way the first part of the data waveform to be retrieved is the last that was stored (see Fig. 1-TTR). Through the change of variables $z^{\prime}=$ $z+c t$ (reference frame moving with $A_{\text {ret }}$ ) and with a kernel (read pulse) $A_{r}(z=0, t)=A_{r 0}(t)=\varepsilon_{r} \exp \left(\imath \theta_{r}\right) \delta\left(t-t_{0}\right)$ the integration, in a reference frame fixed with the fiber, yields:

$$
A_{\text {ret }}(z, t)=A_{t t r} A_{d 0}^{*}\left(\frac{z}{c}+t+t_{0}\right) \exp \left[-\Gamma_{B}\left(\frac{z-L}{c}+t\right)\right] .
$$

where $A_{t t r}=\eta_{B} \Gamma_{B} \varepsilon_{w} \varepsilon_{r} \exp \left[l\left(\theta_{r}+\theta_{w}\right)\right]$. By comparing the latter expression with the data waveform, $A_{d}(z, t)=A_{d 0}(z / c-t)$, and considering that the $A_{t t r}$ is a complex constant (i.e. an amplitude scaling and phase shift) and $t_{0}$ is a delay, it is clear that true time reversal has been achieved. Let us recall that the characteristics of time reversal are: a) the reversal of the propagation direction; b) the reversal of the wavefront envelope and c) the phase conjugation of the envelope, i.e. to achieve the following transformation of a wave:

$$
A(z, t) \exp [\imath(\omega t-k z)] \rightarrow A^{*}(z,-t) \exp [l(\omega t+k z)] .
$$

Two conditions are certainly matched in Eq. (10), in fact: b) the argument of the function $A_{d 0}$ is reversed $(t \rightarrow-t)$; c) the envelope is the complex conjugate of the input data waveform. Condition a) is also matched because the wavevector direction is absolutely reversed. It must be observed that the reversed optical wave has a slightly different frequency $\left(\omega_{d} \rightarrow \omega_{r e t}\right)$, so the wavevector is not simply reversed as in Eq. (11), but rather transformed as $k_{d} \rightarrow-k_{\text {ret }}$. Nonetheless the relative difference in frequency and wavenumber is extremely small (of the order of $\left(\omega_{\text {ret }}-\omega_{d}\right) / \omega_{d}=\Delta n / n \simeq 5 \cdot 10^{-4}$ ).

Finally, let us remark that the obtained true time reversal phenomenon is affected by a distortion, represented by the exponential term of Eq. (10). In fact, the acoustic wave decay is not compensated during the reading process, because the leading edge of the data waveform, that was the first waveform section to be stored, is the last to be read, and so it accumulates a larger decay. However, this fact does not affect the fidelity, because the exponential decay time is a deterministic parameter of the fiber and so it can be compensated by a post-processing.

4) To conceive the arbitrary waveform time integration it is necessary to slightly modify the conditions, though the concept can be still understood with the model presented above. In fact, first a DBG can be created along the entire fiber, on the slow axis, using continuous waves instead of pulses. This means that the stored waveform is $Q$ $(z, t)=\operatorname{rect}(0, L)$, i.e. a rectangular function of length $L$, at all times. Then, the arbitrary function to be integrated is launched on the fast axis. In practice, in this case the kernel of the processing (a rectangular function) is stored first and the signal to be processed is launched later.

Experimental results. All the envisioned DBG-based unconventional processing functions have been experimentally realized.

For the experimental first order time differentiation, the data waveform is a Gaussian pulse (FWHM $7 n s$ ), (blue curve in Fig. 2) and the write pulse is also Gaussian (FWHM 800 ps) launched in a $20 \mathrm{~m}$ long PMF. The kernel (read pulse), ideally the first order 


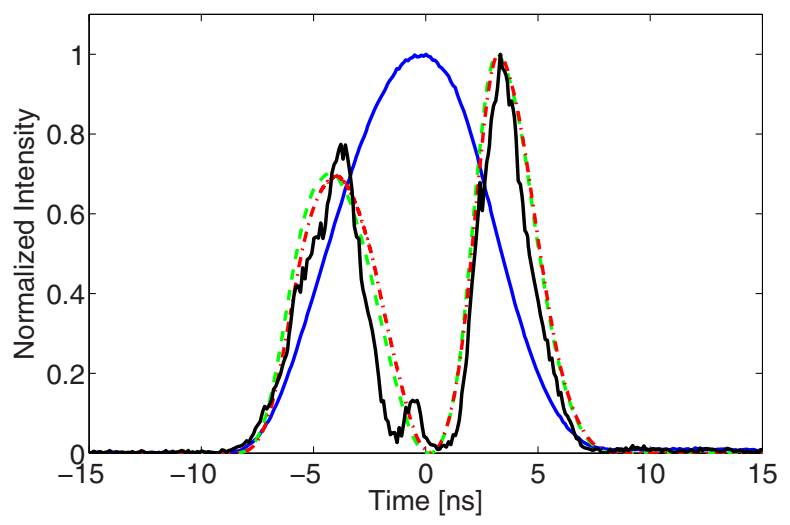

Figure 2 Arbitrary waveform time differentiation: the experimental data waveform (blue curve), the experimental retrieved waveform (black curve), the numerically obtained retrieved waveform when the data waveform is the experimental one (red dashed-dotted curve), the ideal derivative calculated from the experimental data waveform (green dashed curve).

derivative of a Dirac function, is realized through two Gaussian pulses of 800 ps FWHM duration, delayed by 800 ps and $\pi$ phase shifted. The retrieved waveform is shown in Fig. 2. The black, red dashed-dotted, green dashed curves refer respectively to the experimental result, the simulation through Eqs. (1-5) in which the boundary condition are the experimentally detected data waveform, and the exact derivative of the experimental data waveform. The agreement is good, though the first part of the pulse is more distorted: this is to be imputed to the fluctuations of the fiber birefringence that cause a shift in the Brillouin frequency; these effects are particularly large when the DBG has a large spatial extension, as in this case (more than $1 \mathrm{~m})$.

Besides the model provided in the Theoretical Foundations, it might be useful to provide also an intuitive explanation, in terms of the signal backscattering; in fact, the DBG can be regarded as a weak FBG that reflects the read pulse. In the case of the time differentiation, the reflected signal (retrieved waveform) is made by two signals that are phase shifted by $\pi$ radiants. Therefore in the region where they overlap a destructive interference occurs and the first order derivative of the data waveform is achieved. This explanation also highlights another fundamental property of the DBGs, i.e. that they coherently backscatter (i.e. process) the incoming signals.

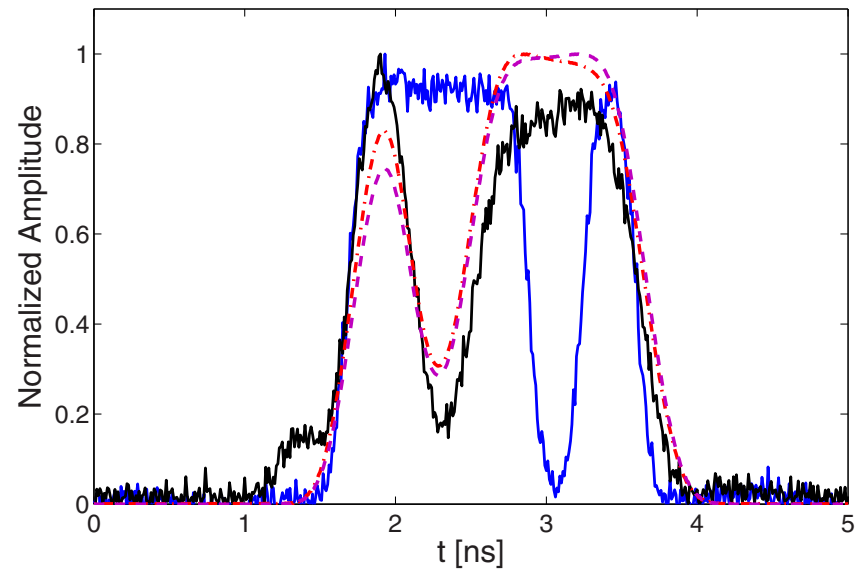

Figure 3 True time reversal: the experimental data waveform (blue curve), the experimental retrieved waveform (black curve), the numerically obtained retrieved waveform when the data waveform is the experimental one without (red dashed-dotted curve) and with (magenta dashed curve) the exponential post-correction.
The data waveform for time reversal, shown in Fig. 3 with a blue curve, is a sequence of eight Gaussian pulses (400 ps FWHM); the write pulse and read pulse are also Gaussian (400 ps FWHM) launched on orthogonal SOPs. The experimentally reversed wave (black curve) is compared to the simulated retrieved waveform without (red dashed-dotted curve) and with (magenta dashed curve) an exponential post-correction (needed as previously remarked); the agreement is good. A certain degree of distortion in the output waveform, that includes a low extinction ratio, changes in pulse intensities and in rise and decay times, can be expected because the write and read pulsewidths used in the experiments were comparable to the sequence pulses. This is better shown in the Supplementary Information. Moreover, additional distortion can arise due to temperature and birefringence changes along the fiber, which cause a shift of the Brillouin gain and of the peak reflectivity wavelength with substantial reflected amplitude change.

As explained in the Theoretical Foundations, in the experimental setup for all-optical signal integration the kernel, a rect function, is written first, by creating a DBG along a $42 \mathrm{~cm}$ long PMF by means of two continuous waves (data waveform and write pulse) In Fig. 4 the read pulse to be integrated (blue curve) is made by two 800 ps FWHM Gaussian pulse, launched along the other orthogonal principle axis. The experimental retrieved waveform (black curve) corresponds to the temporal integration of the read pulse. The comparison between the experimental retrieved waveform (black curve), the retrieved waveform obtained through numerical integration (red dasheddotted curve) and the ideal retrieved waveform (green dashed curve), the latter two using the experimental read pulse, shows a very good agreement. Note, in particular, that the integration is coherently performed over the optical field; in fact, by integrating two in-phase optical pulses (second step in Fig. 4), the detected output power is four times the integration of a single pulse (first step in Fig. 4).

\section{Discussion}

Let us highlight the main peculiarities which make this technique superior to others in realizing signal processing functions, as well as the realized and the perspective applications of the DBGs.

As already mentioned all types of signal processing (calculus and time reversal) can be achieved with the same setup, by simply changing the input signals. Moreover, the processing is coherent, i.e. it operates on the amplitude and the phase of the signals. In the calculus, the DBGs results are similar to those achieved by $\mathrm{FBGs}^{16}$; this can be clearly understood because, as mentioned, the DBGs are actually weak FBGs. The crucial advantage of the DBGs is that the

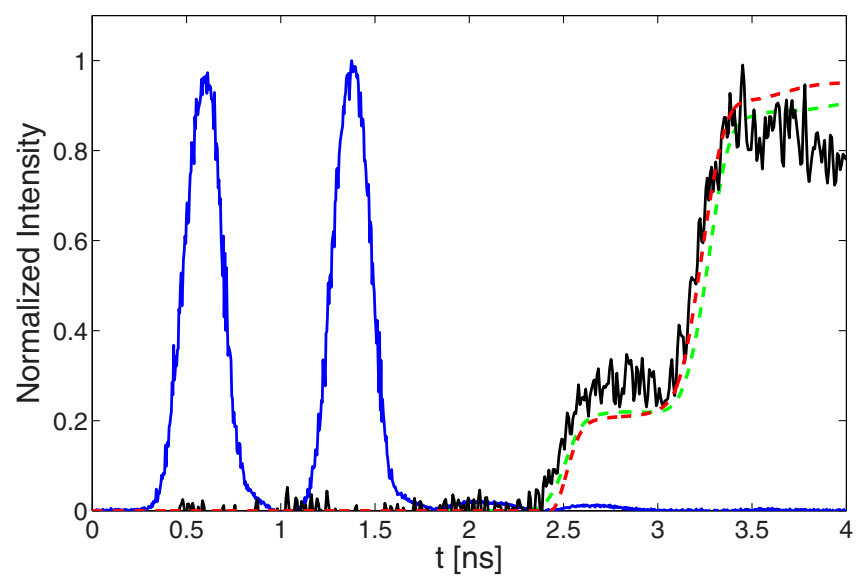

Figure $4 \mid$ Arbitrary waveform time integration: the experimental read pulse (blue curve), the experimental retrieved waveform (black curve), the numerically obtained retrieved waveform when the data waveform is the experimental one (red dashed-dotted curve), the ideal integral calculated from the experimental read pulse (green dashed curve). 


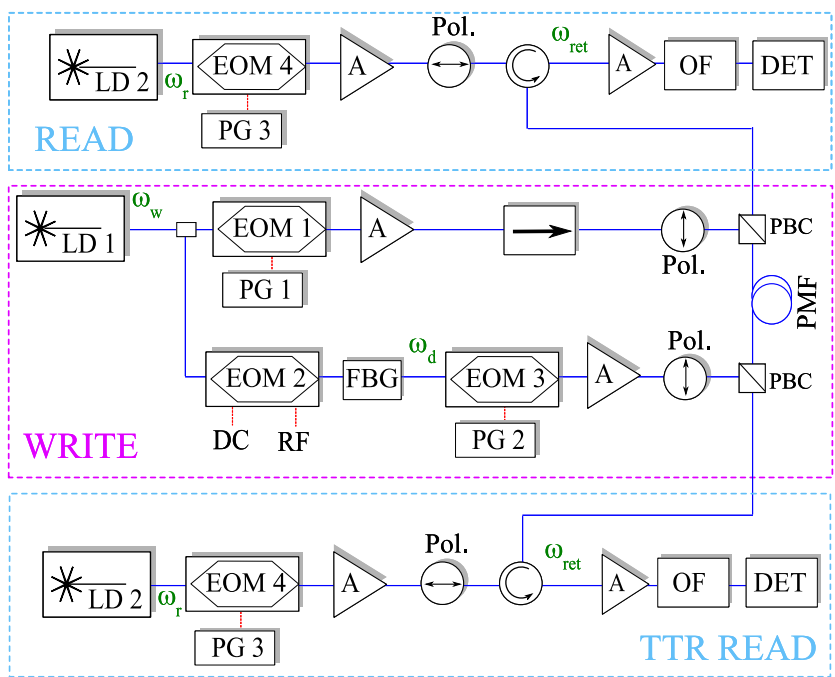

Figure 5 | Experimental setup. LD: laser diode. EOM: electro-optic modulator. PG: pulse or pattern generator. FBG: fiber Bragg grating. A: optical amplifier (EDFA). OF: optical filter. Pol.: polarizer. PBC: Polarization beam combiner. PMF: PM fiber.

grating length can be flexibly tuned, so as to properly adapt to ultrawideband (for the time differentiation) or to long (for the time integration) pulses. In fact, it must be noted that the DBG bandwidth is inversely proportional to its length which is finally dictated by the data waveform and write pulse duration ${ }^{17}$; so there is no bandwidth limitation in principle. However, a practical limitation stems from the fact that by shortening the pulsewidths the corresponding pulse peak power must be increased to maintain the DBG reflectivity and eventually the signal to noise ratio at reasonably large values. The replication of the experimental results in the range of ps pulses, would require peak powers of the order of some $\mathrm{kW}$, which can still be safely handled in optical fibers, but require high power lasers. Moreover, the TTR is a feature that cannot be achieved through FBGs, because it requires a phase conjugation. In fact, all the previous realizations of the time reversal in photonics were based on a phase conjugation phenomenon, like spectrally decomposed-wave mixing $^{18,19}$, spectral hole-burning ${ }^{20}$ and spectral holography ${ }^{21}$. On this regard, let us remark that the DBG-based true time reversal setup, based on fiber optics, presented here is by far much simpler in comparison to the mentioned examples and it is also faster.

The field of application of DBGs in general and of the presented processing functions is, potentially, extremely wide.

First and higher order arbitrary waveforms time differentiation is a very important technique for ultra-wide band radio communications $^{22}$, where the pulse forming with first and higher order time differentiation of short Gaussian pulses is still a difficult task to achieve.

As for TTR, this effect has the intrinsic property to overcome the limitations of wave propagation in highly scattering media, as shown in acoustic science ${ }^{23}$, where true time reversal enabled enhanced therapeutic treatments (ultrasonic lithotripsy and hyperthermia) ${ }^{24}$.

The extension of that concept to the radio science ${ }^{25}$ have also shown a great potential to enhance $\operatorname{radar}^{26}$ and radio communications ${ }^{27}$. The main limitation of the recalled results in electromagnetism, that are based on electronic analog-to-digital conversion and numerical time reversal, is the bandwidth. The proposed DBG scheme can further extend true time reversal techniques to ultrawideband microwave signals.

Finally, the TTR setup proposed here overcomes several of the limitations of the holographic phase-conjugation methods ${ }^{28,29}$ that enabled highly focusing of optical beams in in-vivo tissues. In particular, the holographic methods suffer from the fact that they are not real time techniques. The fields of perspective application can be identified in all those requiring the focusing of optical beams in turbid media, a very typical problem in the medical treatments, like the photodynamic therapy ${ }^{30}$ and the optogenetics ${ }^{31}$.

Eventually, the manipulation of DBGs has a great potential in sensing applications, as recently shown in Ref. 17 and in microwave-photonics where the generation of localized DBGs can enable the realization of very flexible microwave-photonic notch filters ${ }^{11}$, with the unique property of working in the optical coherent regime, since DBGs are phase-conjugating reflectors.

The main limitation of the technique is, as reported above, the very high peak power to be used when short pulses are involved. However this limitation could be relaxed at least by an order of magnitude by using highly nonlinear Brillouin fibers ${ }^{32}$ or photonic crystal fibers ${ }^{8}$ or waveguides $^{33,34}$.

In conclusion, in this paper, unconventional all-optical processing functions, which exploit the generation and manipulation of dynamical Brillouin gratings, have been theoretically predicted, experimentally demonstrated and numerically confirmed. The setup is conceptually very simple and the experimental realization is compact and based on a limited amount of devices. In particular evidence of three processing functions, namely first order time differentiation, time integration and true time reversal, has been given.

As it has been demonstrated that the writing and reading processes are convolutions between optical-optical and optical-acoustic waveforms, so other complex filtering operations can be implemented by using proper kernel pulses. Moreover, all-optical Boolean operations, like AND and NOT gates, on digital optical data can be envisioned thus enabling the construction of all others logical operations.

The processing functions have been entirely realized in optical fibers and therefore can be seamlessly integrated in an optical network with minor insertion losses. The results presented therefore indicate that DBGs can have many potential applications in photonics but also in radio science and medicine.

\section{Methods}

The study of DBGs entailed three approaches: theoretical, numerical and experimental.

The analytical approach is one of the results of this work and for this reason it has been already presented in the Results section. Its strength is that to be able to conceive all the processing functions demonstrated in this work. This stems from the fact that input pulses considered in the model are ideal distribution functions, which make the calculations of the convolution integrals 6, 8, 9, 10 very straightforward. All steps of the integration are provided in the additional information document.

However, the ideal assumptions of the theoretical analysis (where the kernels are distribution functions, and no SPM or SpBN occur) cannot be realized experimentally. So, the validity of the theoretical analysis is also checked through the numerical integrations using the experimental detected input pulses.

Eqs. (1-5) have been numerically integrated through a split-step Fourier propagation algorithm, in which the integration step is carried out in time. The details about the governing equations, including the parameters used in the simulations, are given in the additional information document. Besides confirming all the theoretical and experimental results, the numerical integrations are fundamental to understand how all processing functions can be achieved through simple modifications of the same experimental setup and to identify the experimental features stemming from not ideal conditions. For example, from the numerical results it is found that the SPM only modifies the phase of the colliding pulses and so its effect can be accounted by the arbitrary phases coefficients introduced in the analytical model. As for the SpBN, its contribution is found to be very small. All these issues are better explained in the additional information document.

It is remarkable, that the results of the numerical solutions, and of many of the experiments, are in excellent agreement with the ideal results. On this regard the choice of the processing pulse duration is fundamental. Very broad write and read pulses result into a broadening of the retrieved pulse, thus introducing a distortion. The price to paid for that is an increase in the pulse peak power which is typically larger than $10 \mathrm{~W}$.

As for the experiments, in Fig. 5, a general, simplified setup is shown. For the writing process, the output of a laser diode (LD 1), operating at $\omega_{w}$ is split in two branches. On the first branch the signal is modulated (EOM 1), and amplified (Erbium doped fiber amplifier - EDFA) to generate the write pulse. On the second branch two first-order sidebands at the Brillouin frequency shift are generated by modulation (electro-optic modulator - EOM 2). A complete suppression of the carrier was realized; the higher frequency sideband is filtered by a fiber Bragg grating (FBG) 
and directed to EOM 3 to produce the data waveform, also amplified by an EDFA to peak levels in the range of hundreds of $\mathrm{mW}$. The two waves are linearly polarized and aligned with the slow axis of the PMF. The read pulse is generated using a different laser diode (LD2), that is modulated by EOM 4 and amplified by an EDFA. The read pulse is then linearly polarized and aligned to the fast axis of the PMF (fast axis). A second laser diode is needed because the frequency shift between slow and fast PMF axis is several tens of $\mathrm{GHz}$ and this laser diode needs to be spectrally positioned at $\omega_{r}$. As shown in Fig. 5, the read pulse can be launched following the write pulse or the data waveform; in the latter case, the true time reversal setup is realized. Finally, the retrieved waveform is optically amplified and filtered (to limit the ASE noise) before the detection.

1. Song, K. Y., Zou, W., He, Z. \& Hotate, K. All-optical dynamic grating generation based on Brillouin scattering in polarization maintaining fiber. Opt. Lett. 33 926-928 (2008).

2. Nikles, M., Thévenaz, L. \& Robert, P. A. Brillouin gain spectrum characterization in single-mode optical fibers. J. Lightw. Tech. 15, 18421851 (1997).

3. Bao, X., Webb, D. J. \& Jackson, D. A. 32-km distributed temperature sensor using Brillouin loss in optical fiber. Opt. Lett. 18, 15611563 (1993).

4. Song, K. Y., González Herráez, M. \& Thévenaz, L. Observation of pulse delaying and advancement in optical fibers using stimulated Brillouin scattering. Opt. Expr. 13, 82-84 (2005).

5. Zhu, Z., Gauthier, D. J. \& Boyd, R. W. Stored Light in an Optical Fiber via Stimulated Brillouin Scattering. Science 318, 1748-1750 (2007).

6. Thévenaz, L. et al. Slow light fiber systems in microwave photonics, In Photonics West - Advances in Slow and Fast Light IV. San Francisco, California, USA. Volume 7949(79490B) (2011).

7. Zadok, A., Zilka, E., Eyal, A., Thévenaz, L. \& Tur, M. Vector analysis of stimulated Brillouin scattering amplification in standard single-mode fibers. Opt. Expr. 16, 21692-21707 (2008)

8. Cao, Y., Lu, P., Yang, Z. \& Chen, W. An efficient method of all-optical buffering with ultra-small core photonic crystal fibers. Opt. Expr. 16, 14142-14150 (2008).

9. Kalosha, V. P., Li, W., Wang, F., Chen, L. \& Bao, X. Frequency-shifted light storage via stimulated Brillouin scattering in optical fibers. Opt. Lett. 33, 2848-2850 (2008).

10. Dong, Y., Bao, X. \& Chen, L. Distributed temperature sensing based on birefringence effect on transient Brillouin grating in a polarization maintaining photonic crystal fiber. Opt. Lett. 34, 2590-2592 (2009).

11. Sancho, J. et al. Tunable and reconfigurable multi-tap microwave photonic filter based on dynamic Brillouin gratings in fiber. Opt. Expr. 20, 6157-6162 (2012).

12. Chin, S. \& Thévenaz, L. Photonic Tunable Delay Lines in Optical Fibers. Laser \& Phot. Rev. 6, 728-734 (2012)

13. Boyd, R. W., Rzążewski, K. \& Narum, P. Noise initiation of stimulated Brillouin Scattering. Phys. Rev. A 42, 5514-5520 (1990).

14. Dodin, I. Y. \& Fisch, N. J. Storing, retrieving, and processing optical information by Raman backscattering in plasmas. Phys. Rev. Lett. 88, 165001 (2002).

15. Dodin, I. Y. \& Fisch, N. J. Dynamic volume holography and optical information processing by Raman scattering. Opt. Comm. 214, 83-98 (2002).

16. Azaña, J. Ultrafast analog all-optical signal processors based on fibergrating devices. IEEE Phot. Jour. 2, 359-386 (2010).

17. Song, K. Y., Chin, S., Primerov, N. \& Thévenaz, L. Time-domain distributed fiber sensor with $1 \mathrm{~cm}$ spatial resolution based on Brillouin dynamic grating. J. Lightw. Tech. 28, 2062-2067 (2010).

18. Miller, D. A. B. Time reversal of optical pulses by four-wave mixing. Opt. Lett. 5 , 300-302 (1980).

19. Marom, D., Panasenko, D., Rokitski, R., Sun, P.-C. \& Fainman, Y. Time reversal of ultrafast waveforms by wave mixing of spectrally decomposed waves. Opt. Lett. 25 , 132-134 (2000).

20. Carlson, N. W., Rothberg, L. J., Yodh, A. G., Babbitt, W. R. \& Mossberg, T. W. Storage and time reversal of light pulses using photon echoes. Opt. Lett. 8, 483-485 (1983).

21. Rebane, A., Aaviksoo, J. \& Kuhl, J. Storage and time reversal of femtosecond light signals via persistent spectral hole burning holography. Appl. Phys. Lett. 54, 93-95 (1989).
22. Ghavami, M., Michael, L. B. \& Kohno, R. Ultra Wide-band Signals and Systems in Communication Engineering (Wiley, 2004).

23. Fink, M. Time reversal of ultrasonic fields - part I: basic principles. IEEE Trans. on Ultrason., Ferroel. and Freq. Cont. 39, 555-566 (1992).

24. Fink, M., Montaldo, G. \& Tanter, M. Time-reversal acoustics in biomedical engineering. Annu. Rev. Biomed. Eng. 5, 465497 (2003).

25. Lerosey, G. et al. Time reversal of electromagnetic waves. Phys. Rev. Lett. 92 193904 (2005).

26. Lerosey, G., de Rosny, J., Tourin, A. \& Fink, M. Focusing beyond the diffraction limit with far-field time reversal. Science 23, 1120-1122 (2007).

27. Lerosey, G., de Rosny, J., Tourin, A. \& Fink, M. Timereversal signal processing can boost wireless comms. SPIE Newsroom, http://spie.org/x14501.xml?ArticleID = $\mathrm{x} 14501$ (2007).

28. Yaqoob, Z., Psaltis, D., Feld, M. S. \& Yang, C. Optical phase conjugation for turbidity suppression in biological samples. Nat. Phot. 2, 110-115 (2008).

29. Xu, X., Liu, H. \& Wang, L. V. Time-reversed ultrasonically encoded optical focusing into scattering media. Nat. Phot. 5, 154157 (2011).

30. Brancaleon, L. \& Moseley, H. Laser and non-laser light sources for photodynamic therapy. Lasers Med. Sci. 17, 173186 (2002).

31. Deisseroth, K. Optogenetics. Nat. Meth. 8, 26-29 (2011).

32. Song, K. Y., Abedin, K. S., Hotate, K., González Herráez, M. \& Thévenaz, L. Highly efficient Brillouin slow and fast light using As2Se3 chalcogenide fiber. Opt. Expr. 14, 5860-5865 (2006)

33. Pant, R., et al. On-chip stimulated Brillouin scattering. Opt. Expr. 19, 8285-8290 (2011).

34. Pant, R. et al. Observation of Brillouin dynamic grating in a photonic chip. Opt. Lett. 38, 305-307 (2013).

35. Zou, W., He, Z., Song, K.-Y. \& Hotate, K. Correlation-based distributed measurement of a dynamic grating spectrum generated in stimulated Brillouin scattering in a polarization-maintaining optical fiber. Opt. Lett. 34, 1126-1128 (2009).

\section{Acknowledgements}

The research of Marco Santagiustina is sustained by the University of Padova, through grant CPDA119030, "Signal processing and sensing based on dynamic Brillouin gratings in optical fibers". Sanghoon Chin, Nicolay Primerov and Luc Thévenaz acknowledge the support from the Swiss National Science Foundation through projects 200020-121860 and 200021-134546.The research leading to these results has received funding from EC FET-Open programme (FP7/2007-2011) under grant agreement n. 219299 GOSPEL.

\section{Author contributions}

M.S. conceived the reversal and differentiation experiments. S.C. conceived the integration experiment. M.S. and L.U. carried out the theoretical calculations. S.C., N.P. and L.T. carried out the experiments. L.U. carried out the numerical simulations. M.S., L.U. and L.T. wrote the main manuscript text. S.C., N.P. and L.U. prepared figures. All authors reviewed the manuscript.

\section{Additional information}

Supplementary information accompanies this paper at http://www.nature.com/ scientificreports

Competing financial interests: The authors declare no competing financial interests.

License: This work is licensed under a Creative Common

Attribution-NonCommercial-NoDerivs 3.0 Unported License. To view a copy of this license, visit http://creativecommons.org/licenses/by-nc-nd/3.0/

How to cite this article: Santagiustina, M., Chin, S., Primerov, N., Ursini, L. \& Thévenaz, L. All-optical signal processing using dynamic Brillouin gratings. Sci. Rep. 3, 1594; DOI:10.1038/srep01594 (2013). 